1. Formally audited current practice against national standards from RCPCH's Back to Facing the Future (2013).

2. Held consultation sessions with multidisciplinary PAU team. An assessment proforma including timings, observations, history, examination and predicted outcome was designed to aid data collection.

Areas identified for improvement included:

- Consultant cover at peak times

- Facilities and space

- Waiting times to see a decision maker

- Early discharge/admission planning

- Parental information

Intervention Some improvements were more challenging to tackle than others. We are exploring relocation of PAU to a more fit for purpose area. Consultants are reviewing working patterns to provide cover during peak activity. In the meantime we carried out simpler interventions:

- Following feedback we developed the integrated assessment proforma improving assessment efficiency and focussing professionals towards planning for likely outcome. Prescription boxes and prompts will encourage early interventions (such as antipyretics, dioralyte).

- A junior doctor dedicated to PAU should meet nursing team at shift start ensuring names +/- photos are on the PAU board. A full time trust doctor employed for consistent middle grade PAU cover (although sometimes fills rota gaps).

- Consultants to attend PAU after morning ward rounds and evening handover to discuss $+/$ - review patients.

- Expected waited times standardised o Seen by doctor within 1 $\mathrm{h}$ o Seen by senior within $4 \mathrm{~h}$

- Patient/parent information leaflet distributed, outlining what to expect from a visit to PAU.

Study design Not applicable

Strategy for change We produced Standard Operating Procedures outlining the above expectations. This was presented and e-mailed to all staff. Three months were allowed for changes to be embedded before re-audit.

Measurement of improvement The results of our original and re-audit are summarised in the following Table1:

\begin{tabular}{lll} 
Abstract G567(P) Table 1 & Results of original and re-audit \\
\hline & March 2014 & July 2014 \\
& $n=72$ & $n=96$ \\
\% presenting out of hours (5 pm-9 am) & $47 \%$ & $51 \%$ \\
Seen by doctor within 1 h & $57 \%$ & $75 \%$ \\
Senior review within 4 h & $59 \%$ & $73 \%$ \\
\% of patients seen by a consultant & $12 \%$ & $23 \%$ \\
\% of admitted patients seen by consultant within 24 h & $33 \%$ & $48 \%$ \\
Correct prediction of outcome made at initial assessment & $69 \%$ & $69 \%$ \\
\hline
\end{tabular}

There was an 18\% increase in patients seen within $1 \mathrm{~h}$ and a $14 \%$ increase in patients seen by a senior within $4 \mathrm{~h}$, bringing us closer to national levels. Although consultant input doubled with our initial interventions, it is clear that planned review of consultant working patterns is necessary for us to achieve national standards.

Effects of changes These improvements led to reduced waiting times and earlier decision making and discharge from PAU. There is a tangible sense of improved parental satisfaction, although this is to be formally assessed.

Lessons learnt/Message for others To produce adequate, consistent improvement requires a longer process of workforce planning and service relocation/development. However, we have shown that significant improvements can be made by going 'back to basics'. Formalising and communicating expected standards, more efficient paperwork and maximising medical staffing, has been the mainstay to achieving improvement within our service. It can be difficult to obtain agreement for service developments from all involved, but we found involving all parties early in the process was beneficial.

Please declare any conflicts of interest below

We have no conflicts of interest to declare.

\section{G568(P) SEDATION FOR MRI/CY: A SERVICE IMPROVEMENT PROJECT}

ME Collins, BJ Shields. Paediatrics, University Hospitals Coventry and Warwickshire NHS Trust, Coventry, UK

\subsection{6/archdischild-2015-308599.517}

Context The information for this project was collected from the children's wards at UHCW. Patients attending for elective scans requiring sedation were included. Sedation was administered as per the trust protocol. This protocol is based on current NICE and $\mathrm{BNF}$ guidance.

Weight $<15 \mathrm{~kg}$ - Chloral hydrate sedation

Weight $>15 \mathrm{~kg}$ - Midazolam sedation

Problem We found that sedation of children for elective MRI/ CT scans was insufficient more often than not. This led to failed scans which in turn led to delays in diagnosis, stress for patients and families. In addition this incurred significant financial cost to the Trust due to failed scans and referrals to $\mathrm{BCH}$ for scans under GA.

Assessment of problem and analysis of its causes 60 scans were reviewed over a 6 month period in 2013. 38 children underwent midazolam sedation and 22 were given chloral hydrate.

Chloral hydrate sedation was successful in $90 \%$ of cases, whereas midazolam was only successful in $18 \%$ of cases.

After discussion with the finance department we calculated that failed scans combined with referrals to $\mathrm{BCH}$ was costing the trust c. $£ 70,000$ per year.

Intervention We have discontinued the use of midazolam sedation for MRI/CT scans

The trust is in the process of developing a paediatric GA scan service at UHCW

Strategy for change The initial results of the study were presented to the paediatric department at the monthly clinical audit meeting. After discussion, the decision to stop using midazolam for scan sedation was disseminated amongst community and hospital based paediatricians.

A multi-disciplinary team comprising paediatricians, radiographers, radiologists and anaesthetists was formed. This team is in the process of designing and trialling a GA scan service at UHCW. This will enable us to provide scans to children who would beforehand have probably had 1-2 failed scans and then been referred to $\mathrm{BCH}$. In addition this service may also provide the trust with increased revenue and savings.

Measurement of improvement We now only have children weighing $<15 \mathrm{kgs}$ (i.e. eligible for chloral sedation) attending our unit for elective CT/MRI scans. Children weighing $>15 \mathrm{kgs}$ that require sedation are being referred directly to $\mathrm{BCH}$ for GA scans while we develop our own service. This ensures that they will not need to undergo a probably unsuccessful scan under sedation with us before being referred. 
Effects of changes Discontinuing the use of midazolam sedation has prevented children undergoing a stressful procedure that was unsuccesful in $82 \%$ of cases. This has improved patient care in the form of reducing delays in diagnosis and lowering parental anxiety. It has also saved the trust c. $£ 70,000$ per year in failed scans and referrals to $\mathrm{BCH}$.

Lessons learnt This project has shown me that using a structured approach when considering a problem makes it easier to change systems/processes. Our department knew that midazolam wasn't particularly successful but when you present that as a $82 \%$ failure rate it makes things a lot clearer. Forming an MDT to try to find a solution was also of huge benefit as it very quickly became clear that the radiology department shared our frustrations regarding failure of sedation. This in turn has enabled us to move forward with development of our own GA scan service quite quickly.

Message for others If you feel there is a problem/issue in your department analyse it in a structured way and try to form a solution to the issue rather just presenting a problem

If an MDT needs to be involved ensuring that there are clear goals identified is very important so as to avoid being sidetracked.

\section{G569(P) PATIENT SAFETY IMPROVEMENT PROJECT. PRESCRIPTION OF INTRAVENOUS FLUIDS FOR ADMINISTRATION IN CHILDREN}

S Deepak, T Kwok, T Tinklin. Paediatric Trainee, Royal Derby Hospital, Derby, UK

\subsection{6/archdischild-2015-308599.518}

Context The prescription of IV fluids is a commonplace task on the paediatric ward and its importance is often underestimated. National Patient Safety Agency (NPSA) has issued an alert in 2007 advising clinicians about the risk of hyponatraemia in children receiving intravenous (IV) fluids. Accurate prescription of IV fluids and careful monitoring are crucial in preventing fluid induced hyponatraemia in children.

Hence, a local IV fluid prescription guideline was developed and a patient safety improvement project was set up to ensure that the guideline is adhered to.

Problem A prospective audit was performed in November 2012, looking at all children who were admitted to a busy district general children hospital and prescribed IV fluids over a 2 week period. Prescription of IV fluid rate, type of fluid used, potassium content as well as monitoring of patient's renal function, fluid balance and daily weight were audited.

Assessment of problem and analysis of its causes The results of this audit show that type of fluid, rate of fluid and daily monitoring of UEs are done relatively well. Weight monitoring and fluid balance was poorly done.

Intervention We took few actions to improve the outcome.

Further education of junior doctors, nurses and allied health care professionals was provided.

Fluid prescription chart was updated to act as a visual cue and to enable accurate calculation of fluid rate and renal function monitoring.

Strategy for change Our strategy to improve the compliance to the standards was by focussing on the targeted education package for both medical and nursing team.
We designed an IV fluid teaching booklet with assessments to be carried out by all junior doctors during the departmental induction followed by a seminar every few months.

Awareness regarding the standards was created among nursing colleagues.

Measurement of improvement A similar prospective reaudit was performed in November 2013 on all children admitted over a 2 week period in the same hospital.

Effects of changes The prescription of type of fluid, rate of fluid and daily monitoring of UEs are done relatively well. Prescription of potassium containing fluids, monitoring of daily weights and fluid balance which were badly done on the initial audit with compliance rate of $40 \%, 40 \%$ and $58 \%$ respectively improved to $71 \%, 71 \%$ and $61 \%$ during the reaudit.

Lessons learnt This project highlights the importance of education and enhanced training for staff. We are highlighting the results to all clinician as well as nursing staff during departmental meetings to raise awareness and identify solutions to barriers identified on a regular basis.

Message for others Targeted staff education and training are paramount important in improving the quality of care.

These can be cost effective and by effective organisation, can successfully implement locally.

\section{G570(P) PROJECT PLAY - MEDICAL STUDENTS PROVIDING OUT OF HOURS PLAY FOR PAEDIATRIC PATIENTS}

${ }^{1} \mathrm{H}$ Pearson, ${ }^{1} \mathrm{~L}$ Harlin-Bethel, ${ }^{2} \mathrm{~T}$ Shea. ${ }^{1}$ School of Medicine and Dentistry, Barts and the London, London, UK; ${ }^{2}$ Play Specialist, Barts and the London, London, UK

\subsection{6/archdischild-2015-308599.519}

Context This study examines the effectiveness of Project Play; a collaboration between medical students and health play specialists in order to provide an out of hours play service at The Royal London Hospital (RLH).

Problem There is no out of hours play for children, and there is not enough clinical attachment time for medical students on paediatrics.

Assessment of problem and analysis of its causes The Play Department is currently only able to provide patients with a service Monday to Friday 9-5 pm. The patients need to have access to therapeutic play outside of these hours, however due to staffing we are unable to provide this service.

Students have expressed a high level of interest in paediatrics and a recurrent comment is that there is not enough time in the core module provided for paediatric clinical attachments.

Dissatisfaction is show in this quote from the head of the Human Development module: "Although students have expressed a high level of satisfaction with the Paediatric modules in their Human Development course, a recurrent comment is that there is not enough time in the core module provided for all students. Ours is one of the shortest clinical attachments of UK medical schools."

Intervention All volunteers were required to attend a training day. Then, two volunteering session a week were initiated in October 2014. These ran 6-8 pm on Tuesday and Thursday evenings. Tuesday sessions were aimed at children aged 3-12 years, Thursday 12-18 years. The number of volunteers at each session ranged from 4 to 7 . There is one lead volunteer from the committee at each session. 